\title{
TWO-DIMENSIONAL NUMERICAL SIMULATION OF SALTATING PARTICLES USING GRANULAR KINETIC THEORY
}

\author{
Juan P. Marval \\ Departamento de Energética. Universidad \\ Nacional Experimental Francisco de Miranda. \\ Punto Fijo, Edo. Falcón, Venezuela. \\ jpmarval@unefm.edu.ve
}

\author{
Luis R. Rojas-Solórzano \\ Departamento de Conversión y Transporte de \\ Energía, Universidad Simón Bolívar. \\ Caracas, Venezuela. A.P.89.000. \\ rrojas@usb.ve
}

\author{
Jennifer S. Curtis \\ Chemical Engineering Department, University of \\ Florida, USA. jcurtis@che.edu.edu
}

\begin{abstract}
Most granular flows at environmental conditions are unsteady and exhibit a complex physical behavior. Dune formation and migration in the desert are controlled not only by the flow of saltating particles over the sand bed, but also by turbulent atmospheric airflow. In fact, sediments are transported by the atmospheric airflow within a thin layer only a few centimeters above the sandy surface. These jumping particles reach a maximum sediment mass flux level at a certain delay time (known as the "saturation time") after the initial movement by sliding and rolling begins. Unlike sediment transport in water where the particles are lifted by the turbulent suspension, the saltating particles are kept alive in the layer mainly due to particle-particle and particle-bed collisions. In order to model this Aeolian transport of sand, Jenkins and Pasini [1] proposed a two-fluid model (one-dimensional and steady state) using Granular Kinetic Theory (GKT) to describe the solid-phase stress. The present work extends the original idea of Jenkins and Pasini [1] by using a more robust model of GKT for the kinetic/collisional contributions to the solid-phase stress tensor, together with a friction model activated for sustained contacts between particles. In addition, a standard k- $\varepsilon$ turbulence model for the air and a drag model for the interaction between the phases are employed. A rectangular 2D geometry was chosen with a logarithmic profile for the inlet air velocity, along with an initial amount of sand at rest in the lower part of the simulation domain, resembling the particle saltating flow commonly seen in the vertical middle plane within saltation wind tunnels. This model is validated with experimental data from Liu and Dong [2] and the results given by Pasini and Jenkins [1]. A good estimation for the particle
\end{abstract}

erosion and mass flux in the saltation layer is predicted, even though the profiles of mass flux and concentration within the transport layer are very thin and lower.

Keywords: Saltation layer, Granular Kinetic Theory, CFD.

\section{INTRODUCTION}

Computational Fluid Dynamics (CFD) has recently impacted many fields of research (e.g atmospheric flow, microfluidics, etc.) where before it was unthinkable mainly due to the problems associated to the wide range in length and time scales, as well as the great computational effort necessary to obtain reliable results. Saltating flows are a type of multiphase flow where CFD can now have a great impact. The flow of sediment in the saltating layer is a composite of two phases. Air is the continuum phase or primary phase and sand is the dispersed phase. When the sand particles are considered as an immiscible "fluid", it is possible to use theories developed for two-phase flow. In this case, the dispersed phase of particles and the fluid phase are interpenetrating continua. Recently, the saltation layer has been modeled with mixture models [3,4] neglecting the collisional effects intrinsic to the motion of the particles and the coupling of the particle interactions with the air stream. However, a complete description of sediment transport should consider the three regions present in the phenomenon and the interactions between the regions. The regions are as follows: 1) a bottom region where the particles are at rest (sand bed); 2) a region above the sand bed where the particles are jumping (saltating layer); and 3) a top region where there is only air. Therefore, the mixture model is inadequate, because it does not permit a description of the 
prevailing stratification. Multi-Eulerian models enable the simulation of the three co-existing regions and consider the movement of the cloud of particles with equations that are separate from the gas equations. This approach involves incorporating a description for the solid-phase stress tensorusing the Granular Kinetic Theory (GKT). The present work introduces a numerical model for predicting the flow of sand particles in a saltating state over a sand bed using an Eulerian model for both phases. The gas phase turbulence closure model is the standard $\mathrm{k}-\varepsilon$ model, and the solid-phase stress is described using GKT, with a frictional model which is applied for sustained particle contacts. The mathematical model is implemented in the commercial code ANSYS-Fluent and computed results are compared with experimental data given by [2] and the model predictions given by [1]

\section{NOMENCLATURE}

$\Delta \mathrm{x} \quad \mathrm{x}$-mesh spacing, $\mathrm{m}$

$\Delta \mathrm{y} \quad \mathrm{y}$-mesh spacing, $\mathrm{m}$

$d_{s} \quad$ Particle diameter, $\mathrm{m}$

a Particle radius, $m$

$e \quad$ Particle coefficient of restitution

$\mathrm{Fr} \quad$ Empirical material constant, $\mathrm{Pa}$

$g \quad$ Gravitational constant, $\mathrm{m} \cdot \mathrm{s}^{-2}$

$g_{o} \quad$ Radial distribution function

$l \quad$ length for the averaging process, $\mathrm{m}$

$L \quad$ shortest significant macroscopic length, $m$

$L_{x} \quad$ Domain horizontal dimension, $\mathrm{m}$

$L_{y} \quad$ Domain vertical dimension, $\mathrm{m}$

$n \quad$ Empirical constant in frictional pressure equation

$P \quad$ Empirical constant in frictional pressure equation

$p \quad$ Pressure, $\mathrm{Pa}$

$p_{s} \quad$ Solid pressure or Granular pressure, $\mathrm{Pa}$

$R \quad$ Characteristic length-scale, $\mathrm{m}$

$R_{e} \quad$ Reynolds number

$T$ Time, $\mathrm{s}$

$u * \quad$ Friction velocity

$U_{\infty} \quad$ Free air stream, $\mathrm{m} \cdot \mathrm{s}^{-1}$

$U_{g} \quad$ Air velocity profile, $\mathrm{m} \cdot \mathrm{s}^{-1}$

$\boldsymbol{v} \quad$ Velocity vector, $\mathrm{m} \cdot \mathrm{s}^{-1}$

$\mathrm{V}_{\mathrm{r}}$ : Ratio of terminal velocity of a group of particles to that of an isolated particle

$V \quad$ ratio between the friction velocity and threshold velocity

$x: \quad$ Position vector, $m$

$x: \quad$ Horizontal coordinate

$y$ : $\quad$ Vertical coordinate

\section{Greek symbol}

\section{$\alpha \quad$ Volume fraction}

$\beta \quad$ Interphase drag coefficient, $\mathrm{kg} \cdot \mathrm{m}^{3} \cdot \mathrm{s}^{-1}$

$\phi \quad$ Angle of internal friction

$\gamma_{s} \quad$ Dissipation of granular energy, $\mathrm{kg} \cdot \mathrm{m}^{-3} \cdot \mathrm{s}^{-1}$

$\kappa_{s} \quad$ Conductivity of granular energy $\mathrm{kg} \cdot \mathrm{m}^{-1} \cdot \mathrm{s}^{-1}$

$\lambda_{s} \quad$ Solid bulk viscosity, Pa.s

$\lambda_{m f p} \quad$ Mean free path, $\mathrm{m}$

$\begin{array}{ll}\mu & \text { Viscosity, Pa·s } \\ \mu_{\mathrm{t}} & \text { Turbulent viscosity Pa·s } \\ \rho & \text { Density, } \mathrm{kg} \cdot \mathrm{m}^{3} \\ \delta_{\text {sal }} & \text { Saltation layer thickness, } \mathrm{m} \\ \delta_{\mathrm{BL}} & \text { Boundary layer thickness, } \mathrm{m} \\ \delta_{\mathrm{y}} & \text { Initial thickness of sand at rest, } \mathrm{m} \\ \tau & \text { Stress tensor } \mathrm{N} \cdot \mathrm{m}^{-2} \\ \theta & \text { Shield parameter } \\ \Theta & \text { Granular temperature, } \mathrm{m}^{2} \cdot \mathrm{s}^{-2} \\ \text { Subscripts } \\ \text { fri } & \text { Frictional } \\ g & \text { Gas phase (air) } \\ \mathrm{s} & \text { Solid phase (sand) } \\ p & \text { Particle } \\ \mathrm{kin} & \text { Kinetic } \\ \mathrm{col} & \text { Collisional } \\ \text { sal } & \text { Saltation }\end{array}$

\section{AEOLIAN SALTATION CLOUD MODELS}

The Aeolian process begins when the aerodynamic force (i.e. drag) dislodges a few grains, which roll and slide over the sand bed until they enter into saltation. The entrained grains are accelerated by the wind along their trajectory mainly by the drag force before they once again impact the bed. The particles which impact the bed probably rebound or dislodge other particles, creating a grain chain reaction. The interaction between an impacting grain and the bed is called the "splash process" and this is modeled by the splash function [5] derived statistically from the visualization of particle collisions in experiments [6, 7]. The traveling grains extract momentum from the air and, consequently, the air decelerates and the process of direct aerodynamic entrainment ceases. This negative feedback mechanism reaches equilibrium in the saturation time, and, then, the grains are kept alive in the saltation state only by their collisions with the sand bed. The rate of particle transport becomes constant when equilibrium is reached. The statistical models are based on the four subprocesses previously described: aerodynamics entrainment, the grain trajectory, the grain/collision, and the wind velocity modification. A common feature of all the models is that they consider saltating flow in a free condition of movement without any restrictions or obstacles. This premise makes the statistical model useless for the prediction of the patterns of erosion and/or deposition around solid objects caused by the grain-wall interaction.

\section{CFD MODELS AND GRANULAR KINETIC THEORY}

Currently, a CFD methodology for the appropriate treatment of the sediment transport by Aeolian Saltation does not exist. Recently, Ji, et al. [3] and Alharaf [4] proposed interesting models to simulate the large-scale Aeolian sediment transport, without considering in detail the fluid/particle interaction and without taking into account the collisional interaction between particles as a fundamental parameter in 
their considerations. In both works, they used a homogenous model with a single set of equations, and the disperse phase was modeled with a slip velocity based on a drag function. Although, the effect of particles on the wind profile is weak, the air velocity profile within the Saltation layer is modified by the momentum transfer between the two phases. Therefore, in order to consider the mixture model, it would involve knowing priori the concentration profile, velocity profile and other characteristics of the mixture, that are commonly unavailable. Therefore, although these previous works present significant advantages in the use of CFD to simulate the Saltation layer, they do not take into account some fundamental aspects present in the flow. Ji, et al. [3] utilized the logarithmic and total entrained particle flux profiles observed in experiments in order to include functional relationships into his model. Meanwhile, Alraraf [4] focused his work on an algorithm for treating a moving boundary (erosion-deposition) based on handling an artificial computational domain.

On the other hand, there is some uncertainty about the particle suspension mechanisms present in sheet flows (water) and saltating flows (air), while experimental results only offer some qualitative characteristics of the two types of flows. Jenkins and Hanes [8] implemented, for the first time, a simple one-dimensional and steady two-fluid model using Granular Kinetic Theory for describing the interaction among particles in the sediment transport layer both in water and air. Their results showed a qualitative good estimation of the solids erosion, which suggests that the Granular Kinetic Theory could be used to simulate this phenomenon with success. Jenkins and Pasini [1] extended the previous work by adding an extra term in the gas momentum equations, originating from a second averaging process (given in Hsu et al. [9]) which described an additional mechanism of suspension due to turbulence effects together with the granular pressure gradient. Furthermore, Jenkins and Pasini considered a flow regime between the saltation regime and turbulent suspension regime called the collisional regime yet, at same time, they recognized that this regime had not yet been observed experimentally. Their results gave an overestimation of the sand mass flux in comparison with experimental results from particles in the Saltation state.

However, there is enough evidence from quantitative experimental results and analyses based on the ratio of the particle terminal velocity and the vertical turbulence fluctuation velocity $[10,11]$ demonstrating that in the saltation layer the turbulent suspension is negligible. Instead, the particles are only kept alive in the saltation layer by bed-particle collisions and momentum transfer from the air stream to the particles. In any case, the saltation layer represents a dilute regime where the maximum volume fraction is around $5.10^{-4}$ [2], indicating that the particles in saltation contribute only to the kinetic contribution to the solids viscosity and not the collisional regime like Jenkins and Hanes [8] and Jenkins and Pasini [1] suggest.

\section{MATHEMATICAL MODEL}

Three well-known regions are considered in our simulations, according to the solid volume fraction and velocity fields. One region corresponds to the sand bed, where the solids packing level is high, and the gas and solid velocities are equal to zero. This zone cannot move like a fluid, it is a completely solid region, which is superficially eroded by the wind action. The second region is few centimeters $(0.40 \mathrm{~m})$ above the sand bed (saltation layer), where particles travel quickly, driven by a turbulent shearing airflow. In the third region, outside of the saltation layer, there is only air moving parallel to the bed. In this work, we proposed an Eulerian model (inhomogeneous model) for both phases based on the fact that there are three zones with different concentration and velocities (similar to a stratified flow). Furthermore, the Stokes number for the Saltation layer condition is estimated to be greater than 1.

Researchers agree on the governing equations originally proposed by Anderson and Jackson [12] and later on, by Jackson [13], for the description of the gas-solid flow using an Eulerian approach. However, van Wachen et al. [14] affirm that the difference with the Ishii [15] equations set is negligible on an engineering scale. We have adopted the Ishii equations applied to gas-solid flow by Enwald et al. [16]. These equations assume a suspension of identical spherical particles in an incompressible flow, where particles are characterized by a radius "a", and " $\mathrm{L}$ " is the shortest significant macroscopic length-scale related to the particle motion. . For L $>>$ a, the two scales separate and we can define a process averaging lengthscale, $l$, given by the condition: $a<<l<<L$. This means that the variables are averaged over a region that is larger than the particle size, but smaller than the characteristic system length. Table 1 shows the equations used in this simulation. The governing equations are expressed in Eqs. (1-7). The closure model for the solid phase is based on the Granular Kinetic Theory, which comes from an analogy with the gas kinetic theory developed by Chapman and Cowling [17]. The main idea of this model consists of introducing a granular temperature, which is a measure of the energy level of the particle velocity fluctuations [18]. Through the solution of the granular temperature equation, Eq. (9), it is possible to obtain the pressure for the solid phase, Eq. (10), and the entire transport coefficient, Eqs. (12-18). Lun et al. [19] derived all these expressions considering the inelastic nature of particle collisions, the particles as identical spheres of diameter $d_{s}$, composed of a material of density $\rho_{s}$, the interaction between particles occurring by instantaneous binary collisions, and the granular solid stress tensor resulting from kinetic and collisional contributions.

A constant restitution coefficient is used to take into account the energy dissipated by the inelastic particle collisions. Equation (19) shows the radial distribution function, which can be interpreted as a correction factor that modifies the probability of collision when the solid volume fraction is high (dense regime). Regarding the solid viscosity, the model has 
been improved since the first proposal [19]. Gidaspow [20] and Syamlal et al. [21] implemented some changes in the original equation of Lun et al. [19]; firstly, Gidaspow [20] did not account for the inelasticity of the particles in the kinetic contribution, and secondly, Syamlal et al. [21] neglected the kinetic contribution in the dilute regime. Both equations are very similar, except when the solid volume concentration is low (dilute regime), where Gidaspow's equation gives an inadequate finite value for the solids viscosity, and Syamlal et al.'s equations yield the correct trend in the solids velocity, but underestimate it, as the solids fraction approaches zero. Hrenya and Sinclair [22] resolve this problem by starting from the equation of Lun et al. [19] and introducing a ratio between the mean free path and the characteristic length of the system. Hence, Eq. (14) retains two important features: the solid viscosity is zero when the solid volume fraction is zero, and the solids viscosity is adequately predicted in the dilute regime.

Table 1 Governing equations and closure relations for Gas-Solid Flow.

Fraction volume equation

$\alpha_{g}+\alpha_{s}=1$

Gas continuity equation

$\frac{\partial}{\partial t}\left(\alpha_{g} \rho_{g}\right)+\nabla \cdot\left(\alpha_{g} v_{g}\right)=0$

Gas momentum equations, Ishii [15]

$\frac{\partial}{\partial t}\left(\alpha_{g} \rho_{g} \vec{\nabla}_{g}\right)+\nabla \cdot\left(\alpha_{g} \rho_{g} \vec{\nabla}_{g} \vec{\nabla}_{g}\right)=-\alpha_{g} \nabla p+\nabla \cdot \bar{\tau}_{g}+\nabla \cdot \bar{\tau}_{g}^{\prime \prime}$

$+\alpha_{g} \rho_{g} \vec{g}-\beta\left(\vec{v}_{g}-\vec{v}_{s}\right)+\vec{F}_{\text {Lift }}$

Gas tensor stress

$\bar{\tau}_{g}=\alpha_{g} \mu_{g}\left(\nabla \vec{v}_{g}+\nabla \vec{v}_{g}^{T}\right)-\alpha_{g}\left(\frac{2}{3} \mu_{g}\right) \nabla \cdot \vec{v}_{g} \bar{I}$
Solid continuity equation

(1) $\frac{\partial}{\partial t}\left(\alpha_{s} \rho_{s}\right)+\nabla \cdot\left(\alpha_{s} v_{s}\right)=0$

Solid momentum equations, Enwald et al. [16]

$\frac{\partial}{\partial t}\left(\alpha_{s} \rho_{s} \vec{\nabla}_{s}\right)+\nabla \cdot\left(\alpha_{s} \rho_{s} \vec{\nabla}_{s} \vec{v}_{s}\right)=-\alpha_{s} \nabla p-\nabla p_{s}+\nabla \cdot \overline{\tau_{s}}$

Solid tensor stress

$\bar{\tau}_{s}=\alpha_{s} \mu_{s}\left(\nabla \vec{v}_{s}+\nabla \vec{v}_{s}^{T}\right)+\alpha_{s}\left(\lambda_{s}-\frac{2}{3} \mu_{s}\right) \nabla \cdot \vec{v}_{s} \bar{I}$

Solid pressure

$p_{s}=p_{\text {kin } / \text { col }}+p_{\text {fric }}$

Granular temperature equation

$\frac{3}{2}\left[\frac{\partial}{\partial t}\left(\alpha_{s} \rho_{S} \Theta\right)+\nabla \cdot\left(\alpha_{s} \rho_{S} v_{S} \Theta\right)\right]=\left(-\nabla p_{s} \bar{I}+\bar{\tau}_{s}\right): \nabla \vec{v}_{S}-\nabla \cdot\left(\kappa_{s} \nabla \Theta\right)-\gamma_{s}-3 \beta \Theta$

Where $\Theta=\frac{1}{3}\left\langle{\nabla_{s}^{\prime}}^{2}\right\rangle \quad$ and $\quad \vec{v}_{S}=\left\langle v_{s}\right\rangle+v_{s}^{\prime}$

Kinetic/Collisional pressure by Lun et al. [19]

Solid viscosity

$p_{k i n / c o l}=\alpha_{S} \rho_{S}\left[1+2 \alpha_{S}(1+e) g_{0}\right] \Theta$

$\mu_{\mathrm{s}}=\mu_{\text {kin }}+\mu_{\mathrm{col}}+\mu_{\text {fric }}$

Collisional viscosity, Lun et al. [19]

Kinetic viscosity, Syamlal [21]

$\mu_{c o l}=\frac{4}{5} \alpha_{s}^{2} \rho_{s} d_{p} g_{0}(1+e) \sqrt{\frac{\Theta}{\pi}}$

$\mu_{\text {kin }}=\frac{\alpha_{s} \rho_{s} d_{p} \sqrt{\pi \Theta}}{6(3-e)}\left[1+\frac{2}{5}(1+e)(3 e-1) \alpha_{s} g_{0}\right]$

Kinetic viscosity, Hrenya and Sinclair [22]

$$
\mu_{\text {kin }}=\frac{1}{15} \sqrt{\Theta \pi} \frac{\rho_{S} d_{S} g_{0}(1+e)(3 / 2 e-1 / 2) \alpha_{S}^{2}}{(3 / 2-e / 2)}+\frac{1}{6} \sqrt{\Theta \pi} \frac{\rho_{S} d_{S} \alpha_{S}\left(\frac{1}{2}\left(1+\frac{\lambda_{t l m}}{R}\right)+3 / 4 e-1 / 4\right)}{(3 / 2-1 / 2 e)\left(1+\frac{\lambda_{t l m}}{R}\right)}+\frac{10}{96} \sqrt{\Theta \pi} \frac{\rho_{S} d_{S}}{(1+e)(3 / 2-1 / 2 e) g_{0}\left(1+\frac{\lambda_{t l m}}{R}\right)}
$$


Solid bulk viscosity, Lun et al. [19]

$\lambda_{s}=\frac{4}{3} \alpha_{s} \rho_{s} d_{p} g_{0}(1+e) \sqrt{\frac{\Theta}{\pi}}$

Collisional dissipation of energy, Lun et al.[19]

$\gamma_{s}=12\left(1-e^{2}\right) \frac{\rho_{s} \alpha_{s}^{2} g_{0}}{d_{p} \sqrt{\pi}} \Theta^{3 / 2}$

Conductivity of granular energy, Hrenya and Sinclair [22]

$$
\kappa_{s}=\frac{25 \rho_{s} d_{p} \sqrt{\pi \Theta}}{128}\left[\left(\frac{1}{1+\frac{\lambda_{\text {mp }}}{R}} \frac{8}{\eta g_{0}}+\frac{96}{5} \alpha_{s}\right)\left(\frac{1+12 / 5 \eta^{2}(4 \eta-3) \alpha_{s} g_{0}}{41-33 \eta}\right)+\frac{512}{25 \pi} \eta \alpha_{s}^{2} g_{0}\right] \text { Where } \quad \eta=\frac{1}{2}(1+e)
$$

Conductivity of granular energy, Syamlal [21]

$$
\kappa_{s}=\frac{15 \rho_{s} d_{s} \alpha_{s} \sqrt{\pi \Theta}}{4(41-33 \eta)}\left[1+\frac{12}{5} \eta^{2}(4 \eta-3) \alpha_{s} g_{0}+\frac{16}{15 \pi}(41-33 \pi) \eta \alpha_{s} g_{0}\right]
$$

Frictional viscosity, Shaeffer [24]

$$
\mu_{f r i}=\frac{P_{f r i} \sin (\phi)}{\alpha_{s} \sqrt{\frac{1}{6}\left(\left(\frac{\partial u_{s}}{\partial x}-\frac{\partial v_{s}}{\partial y}\right)^{2}+\left(\frac{\partial v_{s}}{\partial y}\right)^{2}+\left(\frac{\partial u_{s}}{\partial x}\right)^{2}\right)+\frac{1}{4}\left(\frac{\partial u_{s}}{\partial y}+\frac{\partial v_{s}}{\partial x}\right)^{2}}}
$$

Gas Solid Drag coefficient, Syamlal et al. [21]

$$
\beta=\frac{3}{4}\left(0,63+4,8 \sqrt{\frac{V_{r}}{\operatorname{Re}_{p}}}\right)^{2} \frac{\alpha_{S}\left(1-\alpha_{S}\right) \rho_{g}}{V_{r}^{2} d_{p}}\left|\vec{v}_{g}-\vec{v}_{S}\right|
$$

Lift force, Drew and Lahey [27]

$$
\vec{F}_{l i f t}=-0.5 \rho_{g} \alpha_{s}\left(\vec{v}_{g}-\vec{v}_{s}\right) \times\left(\nabla \times \vec{v}_{g}\right)
$$

Turbulent gas viscosity

$$
\mu_{t}=\rho_{g} C_{\mu} \frac{k_{g}^{2}}{\varepsilon_{g}}
$$

Turbulent dissipation energy equation

$$
\begin{aligned}
& \frac{\partial}{\partial t}\left(\alpha_{g} \rho_{g} \varepsilon_{g}\right)+\nabla \cdot\left(\alpha_{g} \rho_{g} \vec{U}_{g} \varepsilon_{g}\right)=\nabla \cdot\left(\alpha_{g} \frac{\mu_{t, g}}{\sigma_{k}} \nabla \varepsilon_{g}\right) \\
& +\alpha_{g} \frac{\varepsilon_{g}}{k_{g}}\left(C_{1 \varepsilon} G_{k, g}-C_{2 \varepsilon} \rho_{g} \varepsilon_{g}\right)
\end{aligned}
$$

The rapid granular flow regime is properly described by the GKT, however when the solid volume fraction is very high (quasi-static flow regime) the GKT underestimates the solid viscosity. In fact, the granular temperature is very low as the result of the high solids packing and weak velocity fluctuations; therefore, this situation can be considered like a fluid-solid phase change. This new regime is characterized by
Radial distribution function, Sinclair and Jackson [23]

$$
g_{0}=\left[1-\left(\frac{\alpha_{S}}{\alpha_{S, \max }}\right)^{1 / 3}\right]^{-1}
$$

Frictional pressure, Johnson et al. [25]

$$
p_{\text {fri }}=\left\{\begin{array}{ccc}
\operatorname{Fr} \frac{\left(\alpha_{s}-\alpha_{s, \min }\right)^{n}}{\left(\alpha_{s, \max }-\alpha_{s}\right)^{p}} & \text { for } & \alpha_{s}>\alpha_{s, \min } \\
0 & \text { for } \quad \alpha_{s}<\alpha_{s, \min }
\end{array}\right.
$$

Terminal velocity, Garside and Dibouni [26]

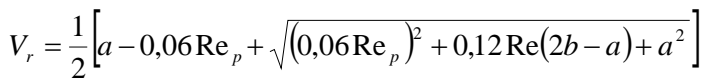

Where:

$$
a=\left(1-\alpha_{s}\right)^{4,14}
$$

$b=\left\{\begin{array}{cc}0,8\left(1-\alpha_{S}\right)^{1,28} & \text { for } \quad \alpha_{S} \geq 0,15 \\ \left(1-\alpha_{S}\right)^{2,65} & \text { for } \quad \alpha_{S}<0,15\end{array}\right.$

Turbulent gas stress tensor

$\bar{\tau}_{g}{ }^{\prime \prime}=\alpha_{g} \mu_{t, g}\left(\nabla \vec{U}_{g}+\nabla \vec{U}_{g}^{T}\right)-\frac{2}{3} \alpha_{g}\left(\rho_{g} k_{g}+\rho_{g} \mu_{t, g} \nabla \cdot \vec{U}_{g}\right) \bar{I}$

Turbulence kinetic energy equation

$$
\begin{aligned}
& \frac{\partial}{\partial t}\left(\alpha_{g} \rho_{g} k_{g}\right)+\nabla \cdot\left(\alpha_{g} \rho_{g} \vec{U}_{g} k_{g}\right)=\nabla \cdot\left(\alpha_{g} \frac{\mu_{t, g}}{\sigma_{k}} \nabla k_{g}\right) \\
& +\alpha_{g} G_{k, g}-\alpha_{g} \rho_{g} \varepsilon_{g}
\end{aligned}
$$

Production of turbulent kinetic energy

$G_{k, g}=\mu_{t, g} S^{2}$

where $\quad S=\sqrt{\frac{1}{2}\left(\frac{1}{2}\left(\nabla v_{g}+\left(\nabla v_{g}\right)^{T}\right)\right)^{2}}$

long-lasting contacts between particles. Johnson and Jackson [28] and Johnson et al. [25] modeled a granular flow following the work of Lun et al. [19], but added the Coulomb friction stress to the solid-phase stress in the momentum equation for quantifying this phenomenon. However, we use the frictional stress Eq. (20) described by [21], originally proposed by Shaeffer [24], instead of the Coulomb equation. Johnson et al. 
[25] proposed the functional form of the semi-empirical equation for the frictional pressure Eq. (21) as a function of the solid volume fraction and maximum and minimum solids packing. The value of the constants $(F r, n$ and $p)$ used in this equation originates from Ocone et al. [29] due to the similarity existing between the particle properties (diameter and density) used in their work and the properties of desert sand. A classical $\mathrm{k}-\varepsilon$ model for turbulence is used as a closure relation for the gas phase, Eqs. (25-29).

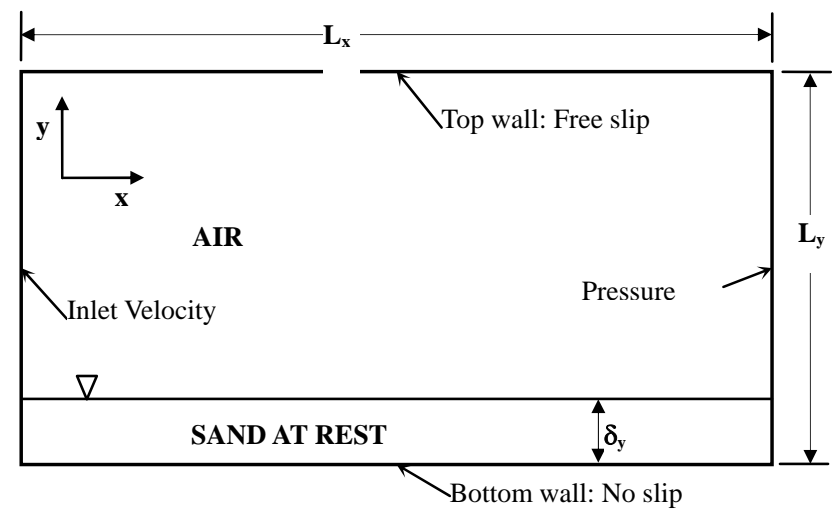

Figure 1 Geometry and boundary conditions for the simulation.

\section{GEOMETRY, GRID, BOUNDARY CONDITIONS AND INITIAL CONDITIONS}

The geometry chosen for the simulation matches the experimental setup and results obtained by Liu and Dong [2], which were made in a saltation wind tunnel with a total length of $37.78 \mathrm{~m}$ and a working cross-section of $0.8 \mathrm{~m} \times 1 \mathrm{~m}$. A twodimensional channel with dimensions $L_{x} \times L_{y}$ was used for representing the computational domain. The vertical dimension $\mathrm{L}_{\mathrm{y}}$ was chosen following two criteria: (a) it should be greater than the experimentally reported boundary layer thickness inside the tunnel; and (b) higher than the height for which the sand mass flux over the bed is almost zero at the maximum test velocity. Thus, based on the previous considerations, $\mathrm{L}_{\mathrm{y}}$ was taken equal to $0.6 \mathrm{~m}$, while $\mathrm{L}_{\mathrm{x}}$ has a value of $1 \mathrm{~m}$.

Gambit v2.2.30 was used as the geometry and grid generator, with a uniform mesh in the $\mathrm{x}$-direction and nonuniform mesh in the $y$-direction. A finer mesh was placed in the vertical direction near to the sand bed, where larger gradients are expected. Meanwhile, a coarser mesh was chosen for the top wall, where a small gradient is expected. The mesh contains 24,000 cells, depicting an aspect ratio with respect to the particle diameter in an approximate range between 5-60.

Although the chosen benchmark experiments have several free stream velocities, we have taken into consideration the highest reported value of $U_{\infty}=18 \mathrm{~ms}^{-1}$ due to good behavior found in the experimental curves of sand concentration and sand mass flux. Equation (30) shows the logarithmical air profile used in the simulation inlet boundary velocity, which was fitted based on air-wind profile data, without sand transport, presented by Liu and Dong [2]. The logarithmical air profile begins just over the sand bed and finishes in the limit of the boundary layer. Below the boundary layer the air velocity is zero, and above is equal to $U_{\infty}$. The origin of the coordinate system is located on the bottom left corner.

$$
U_{g}(y)=\left\{\begin{array}{cc}
0 & 0 \leq y<\delta_{y} \\
a \ln \left(b\left(y-\delta_{y}\right)\right) & \delta_{y} \leq y<\delta_{y}+\delta_{B L} \\
U_{\infty} & y \geq \delta_{y}+\delta_{B L}
\end{array}\right.
$$

For the outlet boundary condition, a gauge pressure equal to zero was specified. Even though the top wall in the saltation tunnel is located $1.0 \mathrm{~m}$ from the bottom, the top boundary condition for the numerical simulation is set at $0.6 \mathrm{~m}$ in order to reduce the number of cells and the simulation time. Hence, a free slip (irrotational flow) condition for both phases is set at $0.6 \mathrm{~m}$ (instead of a no-slip boundary condition). A non-slip condition on the bottom wall for both phases was used. A criterion based on the hydraulic diameter and turbulence intensity was applied for the turbulent boundary conditions at the inlet and backflow outlet, with values of $0.6 \mathrm{~m}$ and $0.05 \%$, respectively.

a)

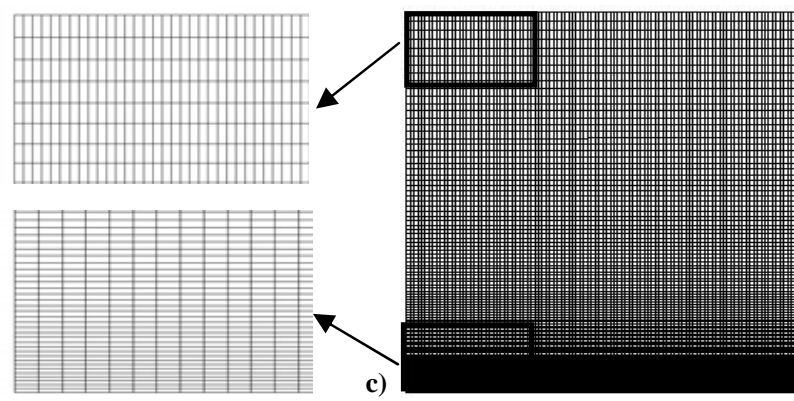

\section{Figure 2 Mesh samples used in the simulation: a) Top left corner, b) Bottom left corner, c) Whole view.}

Transient simulations are strongly dependent on the initial condition, especially in this case where a quasi-steady state in the saltation layer must be achieved in a reasonable interval of time $\left(t_{1}\right.$, see Fig. 3$)$ in order to match experimental data. The experimental procedure used in the wind saltation tunnel indicates that the air flow over the sand bed is fully developed before the initiation of the drag on the particles by the air. This condition was obtained in experiments by covering the sand bed with a geo-textile fabric and then quickly uncovering the bed after the airflow was already fully developed. An equivalent procedure was implemented in ANSYS-Fluent in order to mimic these conditions, i.e., the sand bed remained at rest, while the air flow was fully developed. This condition was obtained by temporarily not solving the equations of granular temperature, volume fraction, and, furthermore, by deactivation of the drag and lift forces. Fully developed air flow was obtained in a real time of only 0.08 seconds with a time step of $1.10^{-3}$ seconds. An initial sand bed with a thickness of $10 \mathrm{~mm}$ 
and a concentration of 0.62 was putting in bottom part of the geometry, which is equivalent to a weight of $16.43 \mathrm{~kg} \mathrm{~m}^{-3}$.

Table 2 Parameters used in the simulation.

\begin{tabular}{lll}
\hline$d_{s}$ & Particle diameter (sand) & $180 \mu \mathrm{m}$ \\
$\rho_{s}$ & Particle density (sand) & $2650 \mathrm{~kg} / \mathrm{m}^{3}$ \\
$\rho_{g}$ & Gas density (air) & $1.225 \mathrm{~kg} / \mathrm{m}^{3}$ \\
$\mu_{g}$ & Gas viscosity (air) & $1.7894 .10^{-5} \mathrm{~Pa} \mathrm{~s}$ \\
$\alpha_{s, \max }$ & Maximum solid volume fraction & 0.63 \\
$\alpha_{\mathrm{s}, \min }$ & Friction Packing Limit (FPL) & $0.5-0.56-0.62$ \\
$e$ & Particle coefficient of restitution & 0.9 \\
$F r$ & Parameter Eq. (21). by [29] & $0.05 \mathrm{~N} / \mathrm{m}^{2}$ \\
$n$ & Parameter Eq. (21). by [29] & 2 \\
$p$ & Parameter Eq. (21). by [29] & 3 \\
$R$ & Characteristic length scale & $0.4 \mathrm{~m}$ \\
$\phi$ & Angle of internal friction & 30 \\
$U_{\infty}$ & Free stream velocity & $18 \mathrm{~m} / \mathrm{s}$ \\
$a$ & Parameter Eq. 30 & $2.2452 \mathrm{~m} / \mathrm{s}$ \\
$b$ & Parameter Eq. 30 & 30303 \\
$\delta_{\mathrm{BL}}$ & Boundary layer thickness & $0.12 \mathrm{~m}$ \\
$\delta_{y}$ & Initial thickness of sand at rest & $0.01 \mathrm{~m}$ \\
$\delta_{s a l}$ & Saltation layer thickness & $0.4 \mathrm{~m}$ \\
$R$ & Characteristic length scale & $0.4 \mathrm{~m}$ \\
$C_{\mu}$ & Parameter Eq. (26) & 0.09 \\
$\sigma_{\mathrm{k}}$ & Parameter Eq. (27) & 1.0 \\
$C_{1 \varepsilon}$ & Parameter Eq. (28) & 1.44 \\
$C_{2 \varepsilon}$ & Parameter Eq. (28) & 1.92 \\
$\sigma_{\varepsilon}$ & Parameter Eq. (28) & 1.3 \\
\hline
\end{tabular}

\section{RESULTS AND DISCUSSION}

Unquestionably, the saltation layer represents a complex problem where the time dependence, small-scale variables (e.g. concentration profiles, mass flux profiles, etc.), and large-scale variables (mass total and mass flux evolution time) are closely interrelated. The strategy followed in our case consisted of obtaining a temporal condition along with a large-scale condition that allowed us to compare the small-scale variables with experimental results. Due to the simulation configuration, there is always a sand mass loss in the domain from the beginning of the simulation until the moment the sand is depleted; therefore, there exists only a short period of time $\left(t_{3}-\right.$ $t_{2}$ ) where a match with experimental data can be made. This condition is called a quasi-steady state, which is shown schematically in Fig. 3, based on the dimensionless total mass and the dimensionless mass flux. From $t=0 \mathrm{~s}$ to $\mathrm{t}=\mathrm{t}_{1}$ the erosion begins over the sand bed; after $t=t_{1}$ is reached, the saturation in the saltation layer and the mass flux is kept constant until $t=t_{2}$. The last part of the process occurs between $t=t_{2}$ and $t=t_{3}$; in this period of time, the mass flux is reduced to zero. The previously described process also occurs in the experimental tests and the characteristic times are mainly a function of the strength of the air-stream. The simulation total time was around of 48 hours for 4 seconds of real time.

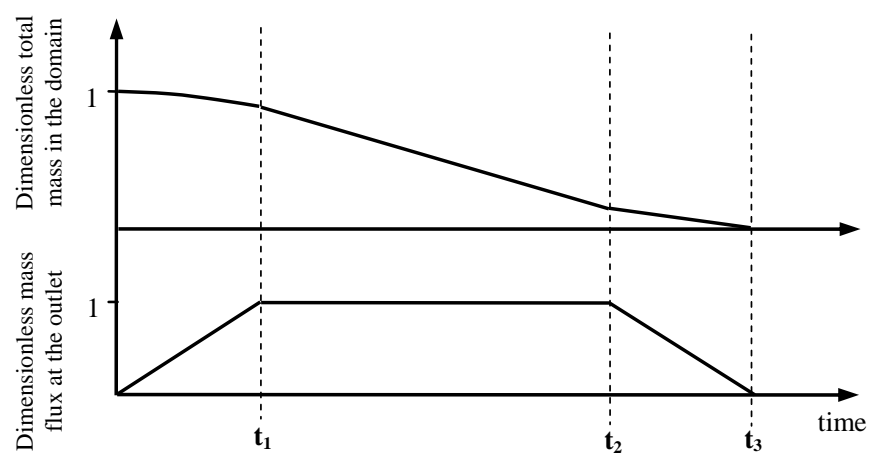

Figure 3 Schematic of the temporal evolution of dimensionless mass flux and dimensionless total mass in the simulation

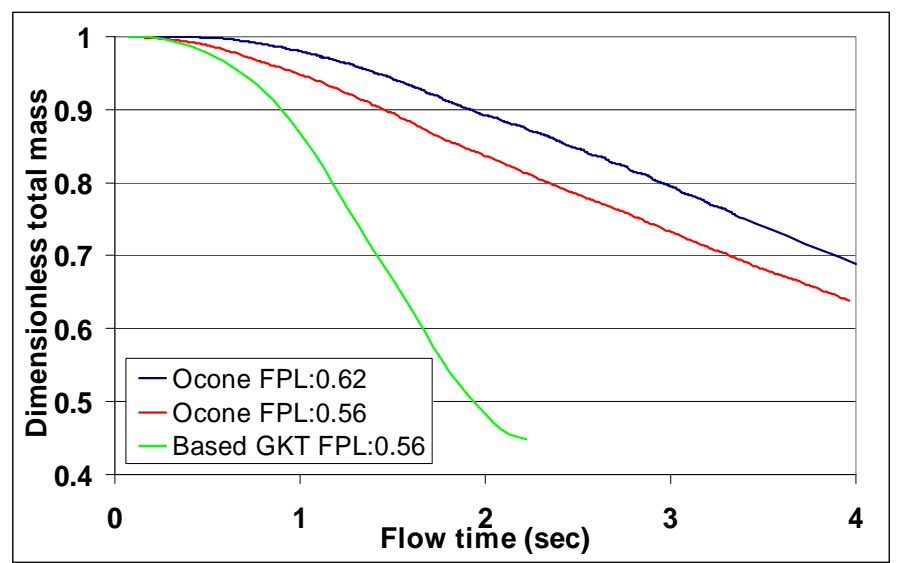

Figure 4 Comparison of temporal evolution of dimensionless total mass from different frictional pressure models. Solid Viscosity and conductivity by Syamlal et al. [21].

Table 3 Parameters of the curves 1, 2, 3, 4 and 5.

\begin{tabular}{|c|l|l|l|}
\hline Curve & Solid viscosity & Solid conductivity & Lift \\
\hline 1 & H and S [22], $\lambda_{\text {mfp }}=20$ & Syamlal et al. [21] & No \\
\hline 2 & H and S [22], $\lambda_{\text {mf }}=5$ & Syamlal et al. [21] & No \\
\hline 3 & H and S [22], $\lambda_{\text {mfp }}=5$ & Syamlal et al. [21] & Yes \\
\hline 4 & H and S [22], $\lambda_{\text {mf }}=5$ & H and S [22], $\lambda_{\text {mfp }}=5$ & Yes \\
\hline 5 & Syamlal et al. [21] & Syamlal et al. [21] & No \\
\hline
\end{tabular}




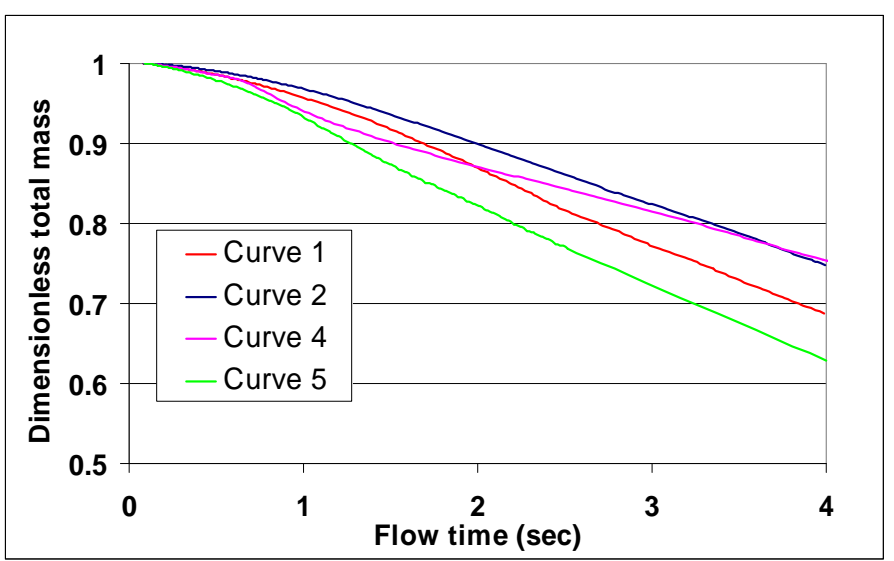

Figure 5 Comparison of temporal evolution of dimensionless total mass from different viscosity models. All curves use a frictional pressure by Ocone et al. [29] FPL=0.5.

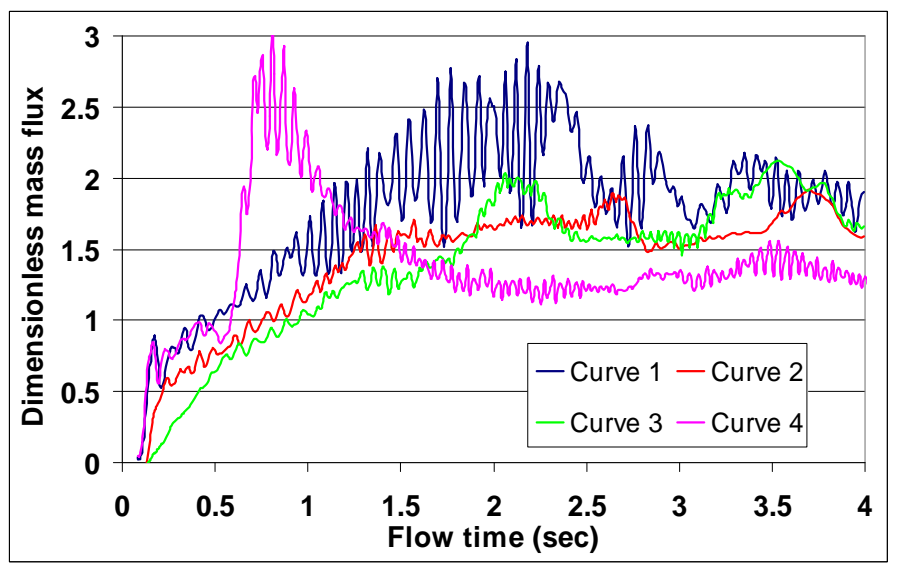

Figure 6 Temporal evolution of dimensionless mass flux at the outlet. All curves use a frictional pressure model based on Ocone et al. [29] FPL=0.5.

\section{Temporal evolution of total mass and mass flux}

The saturation mass flux used to do the dimensionless curves associated with the figure 6 was of $0.744 \mathrm{~kg} \mathrm{~s}^{-1}[2]$. Figure 4 shows the sensitivity of the sand bed to the erosion according to the chosen frictional pressure model. The GKT model uses the kinetic/collisional pressure Eq. (10) in the calculation of the frictional viscosity Eq. (20). This model led to nearly $50 \%$ sand bed erosion in 2 seconds, unlike results based on the frictional pressure model [29] for which the sand bed is hardened due to the high solid pressure added in the solid momentum and granular temperature equations; therefore, the loss of sand mass is smaller in the latter case. We can also see that the variation in the FPL for the model of Ocone et al. [29] produces only changes during the initial period of erosion, but the slope for both curves is the same.

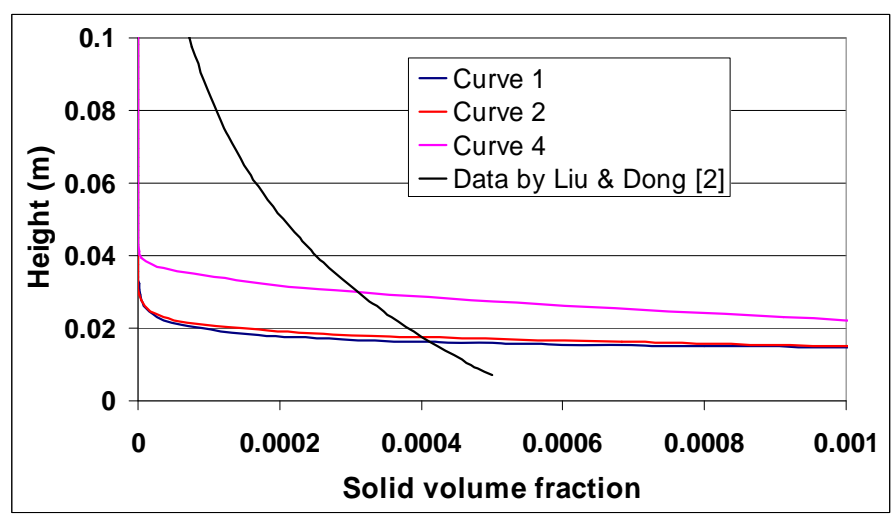

Figure 7 Comparison of the solid volume fraction above the sand bed with experimental data.

The curve 5 in Fig. 5 shows a larger erosion, which corresponds with the Syamlal et al. [21] model for solid viscosity and conductivity, while the curves based in Hrenya and Sinclair [22] for the solid viscosity and conductivity reduce the erosion to between $15 \%-20 \%$ at 4 seconds. The Liu and Dong data [2] show $18.12 \%$ erosion during a period of 4 seconds after total saturation in the transport layer. Previous results indicate that is possible to adjust the model [22] through an appropriate selection of the mean free path to control the erosion. The dimensionless mass flux at the outlet shows sensitivity to the chosen model and parameters, however we can estimate the time $t_{1}$ to be around 2 seconds. The curve 4 in Figure 6 presents the smallest erosion over the sand bed with a dimensionless mass flux around 1.4. The lift force effect is depicted in the simulation results, in curves 3 and 4 in Fig. 6, where the dimensionless mass flux fluctuations are mitigated in comparison with results obtained without considering the lift force (high oscillation).

\section{Solid volume fraction profiles}

The profile of sand concentration within the saltation layer presented in Fig. 7, shows a comparison between the simulation results and experimental data. Liu and Dong [2] data show an exponential decrease of the solids concentration with height over the sand bed, unlike the numerical results, where the transition from the maximum concentration within the sand bed toward zero concentration, outside the saltation layer, happens in a very short vertical length, compared with the saltation layer thickness for this case $(0.40 \mathrm{~m})$. The increase of solid viscosity with the reduction of the mean free path in the Hrenya and Sinclair [19] model improves the predictions of the concentration profile causing more particles to scatter above the sand bed. In Fig. 8, ,a $0.007 \mathrm{~m}$ sand bed thickness is obtained after 4 seconds, using a criterion of 0.5 for the solid volume fraction. 


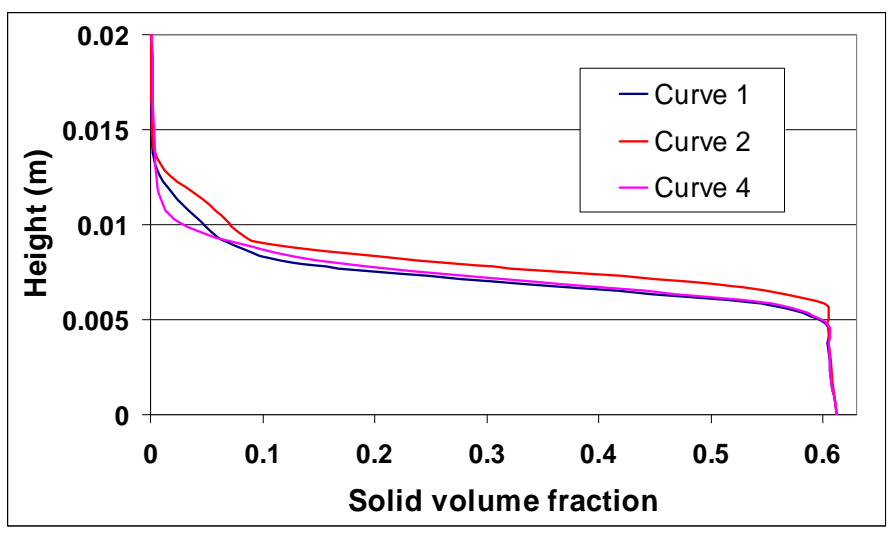

Figure 8 Solid volume fraction within the sand bed.

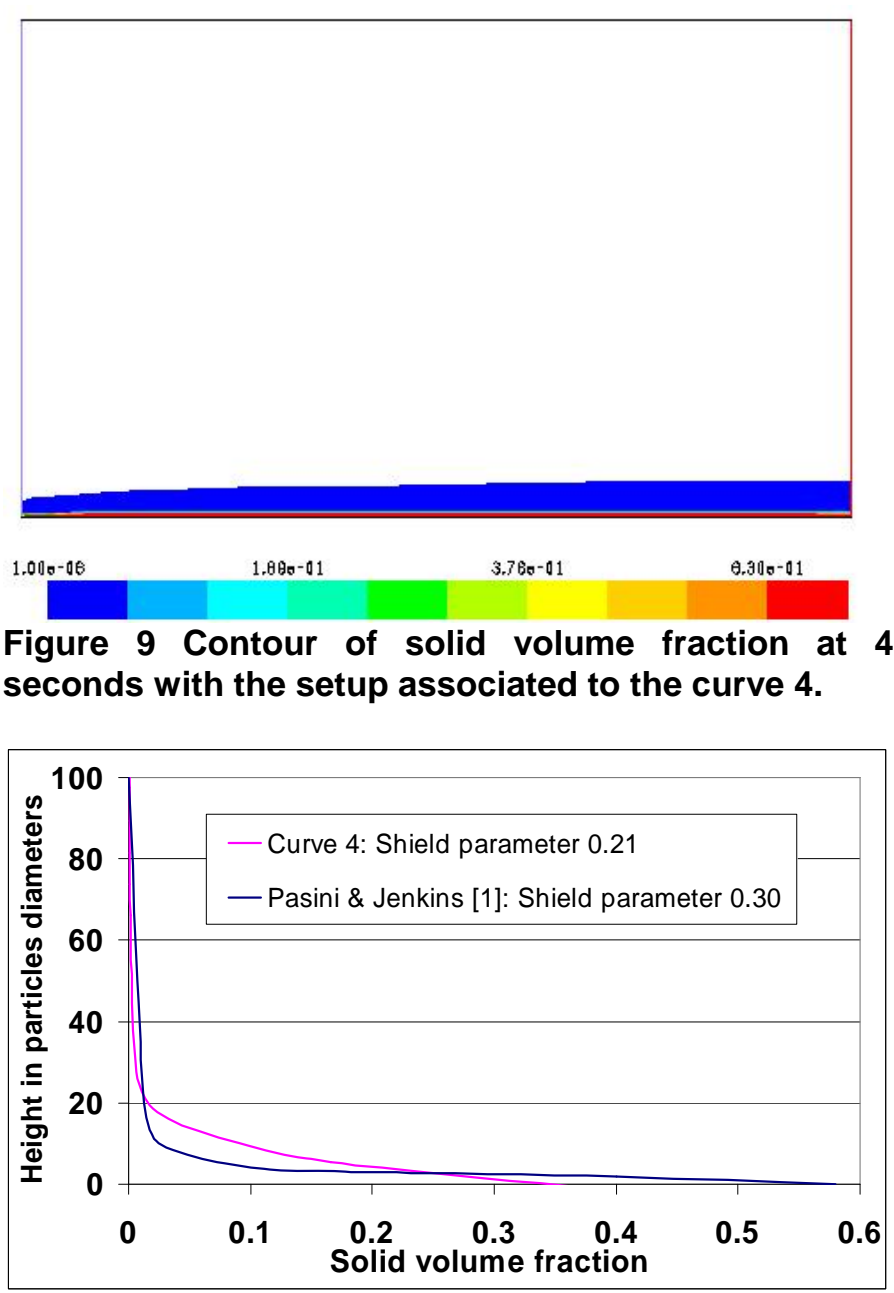

Figure 10 Comparison of solid volume fraction above the sand bed with other numerical results. Height of sand bed: $0.007 \mathrm{~m}$.

Dong et al. [30] gave evidence of the accumulative effect of the mass flux and, consequently, the distribution of sand particles in the saltation state within a wind tunnel. They demonstrated that the mass flux in the saltation layer increases with distance until it reaches a total saturation, after which, the concentration of particles and mass flux remains constant. This behavior can be seen qualitatively in Fig. 9, where the concentration of sand increases over the sand bed along the $\mathrm{x}$ direction.

Figure 10 presents a comparison between our simulations and the concentration profile reported by Pasini and Jenkins [1]. The two curves display a similar behavior with different values for the Shield parameter Eq. (31), a dimensionless measure of the wind velocity strength.

$$
\theta=\frac{u_{*}^{2}}{g\left(\frac{\rho_{S}}{\rho_{g}}-1\right) d_{S}}
$$

\section{Velocities}

A large slip velocity exists between the sand and gas phases (Fig. 11), although the modification in the granular conductivity associated with curve 4 yields less slip in comparison with curve 3 . Figure 12 presents a distribution of the sand $\mathrm{x}$-velocity above the sand bed, with a zero velocity for the sand phase at the top of the sand bed and at the edge of the saltation layer. Also, the sand particles accelerate toward the outlet, where the particle velocity approaches $5 \mathrm{~ms}^{-1}$.

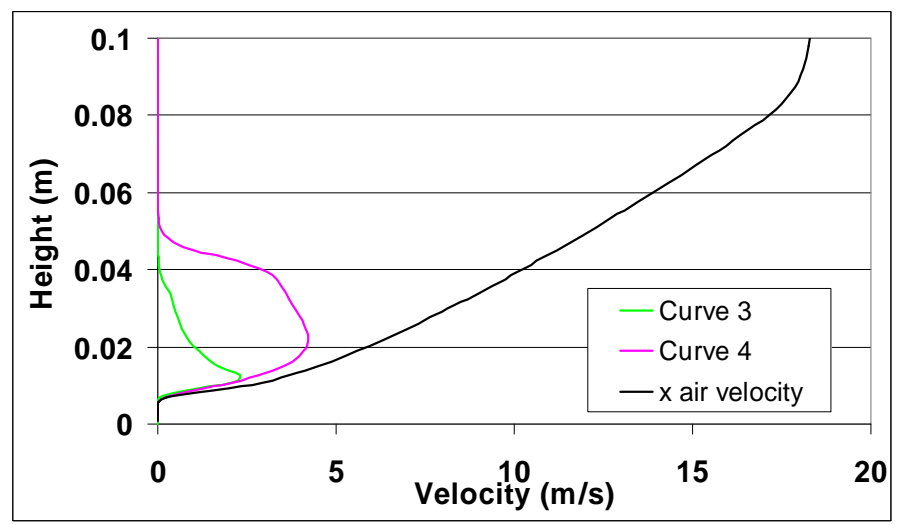

Figure 11 Velocity profiles at the outlet at 4 second

Mass flux profiles

Figure 13 shows the mass flux profiles at the outlet, presenting a similar behavior to that shown by the concentration profiles. The mass flux occurs in a thin layer close to the sand bed, over a thickness of approximately. $0.013 \mathrm{~m}$ for curve 1 , through $0.033 \mathrm{~m}$ for curve 4 . On the scale of Fig. 13, the dimensionless mass flux of Liu and Dong appears like a vertical line with approximately zero dimensionless mass flux, once again indicating that the sand particles in the simulation need to spread away from the sand bed in order to reduce the mass flux near the bed. Curve 4 in Fig. 13 gives the best prediction, which is associated directly with the increase of granular temperature conductivity within the sediment transport layer. 
Fig. 14 shows a similar comparison of mass flux prediction between the present work and that of Pasini and Jenkins [1]. However, the prediction of [1] shows a more uniform mass flux distribution over the height. This is due to the turbulent suspension added by [1] in the $y$-momentum equation. In the present work, the mechanism for suspension is uniquely due to the solid pressure gradient, which is closely related to the variation of the granular temperature.

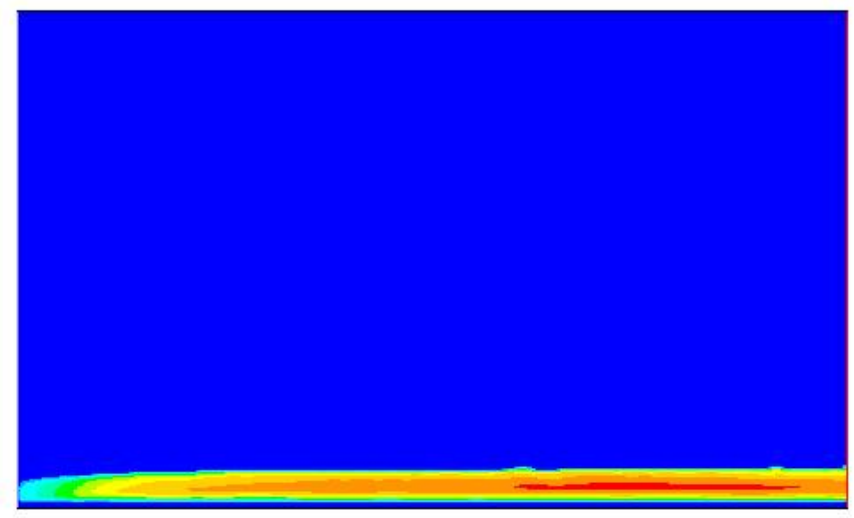

$0.000+00$

$1.500+00$

$3.000+00$

$5.000+00$

Figure 12 Contour of sand x-velocity at 4 seconds with the setup associated to the curve 4.

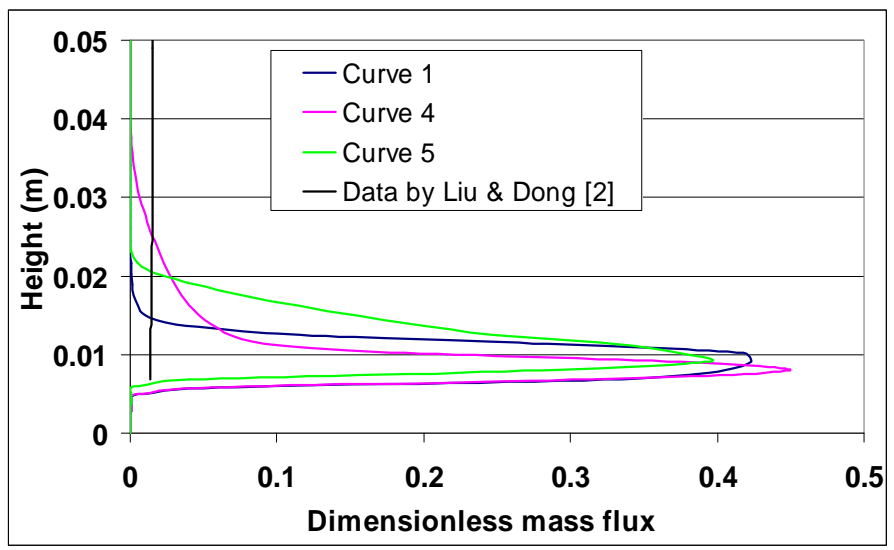

Figure 13 Comparison of dimensionless mass flux in the sediment transport layer with experimental data.

\section{CONCLUSIONS}

From this numerical study, several conclusions may be drawn:

1. The work of Pasini and Jenkins [1] on the Aeolian transport using GKT is extended, considering slightly inelastic particle-particle collisions and incorporating an improved two-dimensional transient model with a frictional sub-model to describe the sustained contacts between particles.

2. The simulation well described the solid-like characteristic of the sand bed, a sand transport layer over the sand bed and an air free flow outside the saltation zone. This results show the ability of our model to describe in future simulations the interaction between the saltation layer and the sand bed with obstacles, and moreover, to obtain the sand and air flow patterns around obstacles, including details about deposition and erosion.

3. Large-scale results overpredict the erosion as indicated by mass fluxes over $40 \%$.

4. Small-scale results show a sediment transport layer that is very thin in comparison with the experimental data.

5. Based on these results, we can affirm that the GKT provides a relatively good description of the saltation layer given the modification of the solid viscosity (due to the kinetic contribution) and conductivity which greatly influence the mass flux, velocities and concentration.

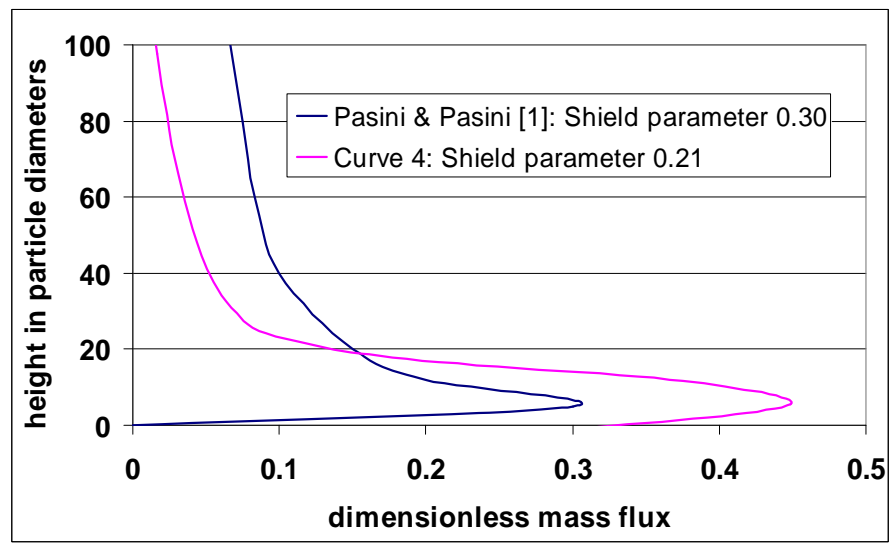

Figure 14 Comparison of dimensionless mass flux above sand bed with numerical results by others. Height of sand bed: $0.007 \mathrm{~m}$.

\section{FUTURE WORK}

The Aeolian sediment transport is influences by many variables and, therefore, it is necessary to perform studies where the influences of many parameters are considered. The model proposed in this work allowed us to analyze the influence of the following parameters on the phenomenon: free stream velocity, slope of the sand bed, particle diameter and sand density. Also, we plan to extend the length of the computational domain in the $\mathrm{x}$-direction in order to analyze the effects of particle accumulation on the mass flux and concentration; this could lead to better comparisons of simulated results with experimental data.

In addition, a more indepth study of the effects caused by the granular pressure and temperature on the particle suspension should be conducted. It is expected that modifications in the granular temperature equation could improve the distribution of sand particles over the sand bed, based on consideration of the dissipation of granular energy and granular conduction. 
Finally, including obstacles like cylinders, flat surfaces and aerodynamic profiles may enrich this study by evaluating their interaction with the sediment transport layer.

\section{ACKNOWLEDGMENTS}

The author Juan P. Marval acknowledges the financial support given by the Fondo Nacional de Ciencia y Tecnologia (FONACIT, S3-2006000474) and by the Universidad Nacional Experimental Francisco de Miranda for the expenses for visiting the University of Florida.

\section{REFERENCES}

[1] Pasini, J.M. and Jenkins, J.T., 2005, "Aeolian Transport with Collisional Suspension,” Phil. Trans. R Soc. A. 363, 16251646.

[2] Liu, X. and Dong, Z., 2004, "Experimental Investigation of the Concentration Profile of a Blowing Sand Cloud," Geomorphology 60, 371-381.

[3] Ji, S.B., Gerber, A.G., Sousa, A., 2004, "A ConvectionDiffusion CFD Model for Aeolian Particle Transport,” Int. J. Numer. Meth Fluids 45, 797-817.

[4] Alhajraf, S., 2004, "Computational Fluid Dynamic Modeling of Drifting Particles at Porous Fences," Environmental Modelling and Software 19, 163-170.

[5] Ungar, J. E. and Haff, P. K., 1987, "Steady-State Saltation in Air," Sedimentology 34, 289-299.

[6] Nalpanis, P., Hunt, J. and Barret, C., 1993, "Saltating Particles Over Flat Bed,” Journal of Fluid Mechanics, 251, 661-685.

[7] McEwan, I. and Willets, B., 1993, "Adaptation of NearSurface Wind to the Development of Sand Transport," Journal of Fluid Mechanics 252, 99-115.

[8] Jenkins, J. T. and Hanes, M., 1998, "Collisional Sheet Flows of Sediment Driven by a Turbulent Fluid," Fluid Mech. 370, 29-52.

[9] Hsu, T. J., Jenkins, J. T., Liu, P. L.-F., 2004, "On TwoPhase Sediment Transport: Sheet Flow of Massive Particles," Proc. R. Soc. A 460, 2223-2250

[10] Sauermann, G., 2001, "Modeling of Wind Blown Sand and Desert Dunes,” Ph. D. Thesis. Universidad de Stuttgart.

[11] Pye, K. and Tsoar, H., 1990, Aeolian Sand and Sand Dunes. London: Unwin Hyman.

[12] Anderson T.B., Jackson R., 1967, "Fluid Mechanical Description of Fluidized Beds,” Ind. Eng. Chem. Fundam. 6, 527-539.

[13] Jackson, R., 1997, "Locally Averaged Equations of Motion for a Mixture of Identical Spherical Particles and a Newtonian Fluid,” Chemical Engineering Science 52, No. 15, 2457-2469.

[14] van Wachen, B.G.M., Shouten, J.C., Krishna, R., den Bleek, C. M. Van and Sinclair, J. L., 2000, "Comparative Analysis of CFD Models of Dense Gas-Solid Systems," AIChE Journal 47 (5), 1035-1051

[15] Ishii, M., 1975, Termo-Fluid Dynamic Theory of TwoPhase Flow, Direction des Etudes et Recherches d’electricé de France, Eyrolles, Paris.
[16] Enwland, H., Peirano, E., Almstedt, A.-E., 1996, “Eulerian Two-Phase Flow Theory Applied to Fluidization," Int. J. Multiphase Flow 22, suppl. 21-66.

[17] Chapman, S. and Cowling, T., 1970, The Mathematical Theory of Non-Uniform Gases,Cambridge Mathematical Library, Cambridge 3rd edition.

[18] Ogawa, S., 1978, "Multitemperature Theory of Granular Material,” Proc. US-Japan Seminar of Continuum Mechanical and Statistical Approaches in the Mechanics of Granular Materials, 208, Gakujutsu Bunken Fukukai, Tokyo.

[19] Lun C. K. K., Savage, S.B., Jeffrey, D.J. and Cherpuniy, N., 1984, "Kinetic Theories for Granular Flow: Inelastic Particle in Couette Flow and Slightly Inelastic Particles in a General Flowfield,” Journal of Fluid Mechanics 140, 223-256. [20] Gidaspow, D., 1994, Multiphase Flow and Fluidization, Academic Press Inc., Boston, 1st edition.

[21] Syamlal, M, Rogers, W. and O’Brien, T. J., 1993, “MFIX Documentation, Theory Guide," Technical Note DOE/METC 94/1004. Morgantown, Wes Virginia.

[22] Hrenya, C., and Sinclair, J., 1997, "Effects of ParticlePhase Turbulence in Gas-Solid Flows," AIChE Journal 43, 853-869.

[23] Sinclair, J., and Jackson, R., 1989, "Gas Particle Flow in a Vertical Pipe with Particle-Particle Interactions," AIChE Journal 35, 1473.

[24] Shaeffer, D G., 1987, "Instability in the Evolution Equations Describing Incompressible Granular Flow”, Journal Differ. Eqs. 66, 19.

[25] Johnson, P., Nott, P., and Jackson, R., 1990, "FrictionalCollisional Equations of Motion for Particle Flows and their Application to Chutes,” Journal Fluid Mechanic 210, 501-535.

[26] Garside, J., and Al-Dibouni, M. R., 1977, "VelocityVoidage Relationship for Fluidization and Sedimentation," Ind. Eng. Chem. Proc. Des. Dev. 16, 206.

[27] Drew, D. A. and R. T. Lahey, 1993, "In Particulate TwoPhase Flow,” Butterworth-Heinemann, Boston, 509-566.

[28] Johnson, P. and Jackson, R., 1987, "Frictional-Collisional Constitutive Relations for Granular Material, with Application to Plane Shearing”, Journal of Fluid Mechanics 176, 67-93.

[29] Ocone, R., Sundaresan, S. and Jackson, R., 1993, "Gas Particle Flow in a Duct of Arbitrary Inclination with ParticleParticle Interactions,” AIChE Journal 39 (8), 1261-1271.

[30] Dong, Z., Wang, H., Liu, X. and Wang, X., 2004, “The Blown Sand Flux Over a Sandy Surface: A Wind Tunnel Investigation on the Fetch Effect," Geomorphology 57, 117127. 